\title{
Assistência multiprofissional em paciente com Atrofia Muscular Espinhal: relato de caso
}

\author{
Multiprofessional care in a patient with Spinal Muscular \\ Atrophy: case report
}

\author{
Atención multiprofesional en un paciente con Atrofia \\ Muscular Espinal: reporte de caso
}

\author{
Mayra da Silva ${ }^{1}$, Sarah Cristina Sato Vaz Tanaka², Virgínia Resende \\ Silva Weffort ${ }^{3}$, Alessandra Bernadete Trovó de Marqui ${ }^{4}$
}

1.Acadêmica do Curso de Graduação em Enfermagem da Universidade Federal do Triângulo Mineiro, UFTM, Uberaba-MG, Brasil. ORCID: https://orcid.org/0000-0001-7708-5231

2.Biomédica, Pós Doutoranda do Programa de Pós-Graduação em Medicina Tropical e Infectologia da UFTM, Uberaba-MG, Brasil. ORCID: https://orcid.org/0000-0003-4466-6093

3.Médica, Doutora, Docente do Departamento de Pediatria da UFTM, Uberaba-MG, Brasil. ORCID: https://orcid.org/0000-0002-2299-0530

4.Biológa, Doutora, Docente da Disciplina de Genética, Departamento de Patologia, Genética e Evolução da UFTM, Uberaba-MG, Brasil. ORCID: https://orcid.org/0000-0003-2361-5174

\begin{abstract}
Resumo
A Atrofia Muscular Espinhal (AME) é uma doença neuromuscular de origem genética, com herança autossômica recessiva, caracterizada pelo declínio progressivo dos neurônios motores e dividida em quatro subtipos: I, II, III e IV, sendo o tipo I o mais grave e corresponde a cerca de $60 \%$ dos casos. Nesse sentido, o objetivo deste estudo é descrever um paciente com AME tipo I com enfoque nos cuidados terapêuticos, relatando a assistência multiprofissional prestada. A paciente nasceu em novembro de 2012 e foi diagnosticada com AME em dezembro de 2013 por meio de exame genético. Desde sua internação no Hospital de Clínicas da Universidade Federal do Triângulo Mineiro, recebe cuidados multidisciplinares das seguintes áreas: Enfermagem, Fisioterapia, Medicina, Nutrição, Pedagogia, Psicologia, Serviço Social e Terapia Ocupacional. Em suma, a assistência multidisciplinar é fundamental e obrigatória para pacientes com essa condição genética, aumentando sua sobrevida e melhorando sua qualidade de vida.
\end{abstract}

Unitermos. Atrofia Muscular Espinhal; Doenças Neuromusculares; Equipe de Assistência ao Paciente

\begin{abstract}
Spinal Muscular Atrophy (SMA) is a neuromuscular disease of genetic origin, with autosomal recessive inheritance, characterized by the progressive decline of motor neurons and divided into four subtypes: I, II, III, and IV, with type I being the most severe and corresponds to about $60 \%$ of cases. In this sense, the aim of this study is to describe a patient with type I SMA with a focus on therapeutic care, reporting the multidisciplinary care provided. The patient was born in November 2012 and was diagnosed with EBF in December 2013 through genetic testing. Since her admission at the Hospital de Clínicas of the Federal University of Triângulo Mineiro, she has received multidisciplinary care in the following areas: Nursing, Physiotherapy, Medicine, Nutrition, Pedagogy, Psychology, Social Work, and Occupational Therapy. In short, multidisciplinary care is essential and mandatory for patients with this genetic condition, increasing their survival and improving their quality of life.
\end{abstract}

Keywords. Muscular Atrophy, Spinal; Neuromuscular Diseases; Patient Care Team 


\section{Resumen}

La Atrofia Muscular Espinal (AME) es una enfermedad neuromuscular de origen genético, con herencia autosómica recesiva, caracterizada por el declive progresivo de las neuronas motoras y dividida en cuatro subtipos: I, II, III y IV, siendo el tipo I el más grave y corresponde a aproximadamente el $60 \%$ de los casos. En este sentido, el objetivo de este estudio es describir un paciente con AME tipo I con un enfoque de atención terapéutica, informando la atención multidisciplinar brindada. La paciente nació en noviembre de 2012 y fue diagnosticada de LME en diciembre de 2013 mediante pruebas genéticas. Desde su ingreso en el Hospital de Clínicas de la Universidad Federal del Triângulo Mineiro, ha recibido atención multidisciplinar en las siguientes áreas: Enfermería, Fisioterapia, Medicina, Nutrición, Pedagogía, Psicología, Trabajo Social y Terapia Ocupacional. En definitiva, la atención multidisciplinar es fundamental y obligatoria para los pacientes con esta patología genética, aumentando su supervivencia y mejorando su calidad de vida.

Palabras clave: Atrofia Muscular Espinal; Enfermedades Neuromusculares; Grupo de Atención al Paciente

Trabalho realizado na Universidade Federal do Triângulo Mineiro/UFTM, Uberaba-MG, Brasil.

Endereço para correspondência: Alessandra Bernadete Trovó de Marqui, Universidade Federal do Triângulo Mineiro/UFTM, Instituto de Ciências Biológicas e Naturais/ICBN, Departamento de Patologia, Genética e Evolução, Disciplina de Genética, Campus I. Praça Manoel Terra 330. CEP 38015-050. Uberaba-MG, Brasil. Fone (34) 3700-6434. E-mail: alessandra.marqui@uftm.edu.br

\section{INTRODUÇÃO}

A Atrofia Muscular Espinhal (AME) é uma doença neuromuscular de origem genética, com herança autossômica recessiva, caracterizada pelo declínio progressivo dos neurônios motores. É classificada em tipos I, II, III e IV, sendo que o I é o mais grave e corresponde a cerca de $60 \%$ dos casos. A idade de início varia de 0-6 meses e a mortalidade é frequente antes dos dois anos de idade. O tipo II costuma manifestar-se entre os 7-18 meses, com expectativa de vida entre $>2$ anos $\mathrm{e}<18$ anos. Os tipos III e IV afetam pacientes com mais de 18 meses e adultos, respectivamente, e possuem expectativa de vida normal ${ }^{1}$. A AME tipo I, também conhecida como Síndrome de Werdnig-Hoffman, é causada por uma mutação nos genes SMN1 e SMN2 (Survival Motor Neuron), localizados no braço longo do cromossomo 5 (5q13) $)^{2-4}$. 
É considerada uma doença rara, pois acomete cerca de 1:6.000 a 1:10.000 nascimentos, com elevada taxa de mortalidade. Os sinais e sintomas observados são comuns a outras neuropatias, o que torna o diagnóstico difícil. Os pacientes apresentam hipotonia, fraqueza muscular, controle insuficiente da cabeça, redução de reflexos ou arreflexia, dificuldade de deglutição, padrão respiratório ineficiente que podem evoluir para insuficiência respiratória, porém, a cognição continua intacta. O diagnóstico pode ser realizado com base no quadro clínico, nos antecedentes familiares e nos exames de eletromiografia e biópsia muscular. No entanto, o padrão ouro para o diagnóstico é uma análise quantitativa de SMN1 e SMN2, usando MLPA (Multiplex Ligation-dependent Probe Amplification) ou qPCR (quantitative Polymerase Chain Reaction $)^{1-4}$.

A AME tipo I é uma doença genética neurodegenerativa progressiva cujo cuidado terapêutico multiprofissional auxilia diretamente no controle dos sintomas, visando a melhora na qualidade de vida do paciente e consequentemente, no desenvolvimento e aperfeiçoamento de práticas baseadas em evidências. Uma pesquisa recente mostrou que a AME tem um impacto significativo na vida dos pacientes e seus cuidadores ${ }^{5}$.

Nesse sentido, o objetivo deste estudo é descrever um paciente com AME tipo I com enfoque nos cuidados terapêuticos, relatando a assistência multiprofissional prestada. 


\section{RELATO DO CASO}

O presente estudo é do tipo relato de caso e foi aprovado pelo Comitê de Ética em Pesquisa do Hospital de Clínicas da Universidade Federal do Triângulo Mineiro (HCUFTM), sob no CAAE 26730819.1.0000.5154. Trata-se de uma paciente de oito anos de idade, admitida no HC-UFTM e diagnosticada com AME tipo I. As principais informações relativas à paciente desde seu nascimento $(11 / 2012)$ até o diagnóstico molecular (12/2013) estão apresentadas na Figura 1.

O exame genético utilizou a metodologia MLPA que revelou nenhuma cópia de SMN1 e três cópias de SMN2. Deste modo, o paciente possui AME tipo $1 \mathrm{~B} / \mathrm{C}^{1}$.

A paciente permaneceu na Unidade de Terapia Intensiva (UTI) Neonatal de julho/2013 a julho/2016. Desde então, ela encontra-se hospitalizada no Setor de Pediatria do HC-UFTM. Durante esse período, ocorreram duas tentativas de alta hospitalar (março/2014 e agosto/2016), porém, sem sucesso.

Os cuidados multidisciplinares recebidos pela paciente desde sua admissão no HC-UFTM são apresentados no Quadro 1. 
Figura 1. Paciente, M.N.F., 8 anos, admitida no Hospital de Clínicas da Universidade Federal do Triângulo Mineiro (HC-UFTM), diagnosticada com Atrofia Muscular Espinhal (AME) e características sobre o diagnóstico.

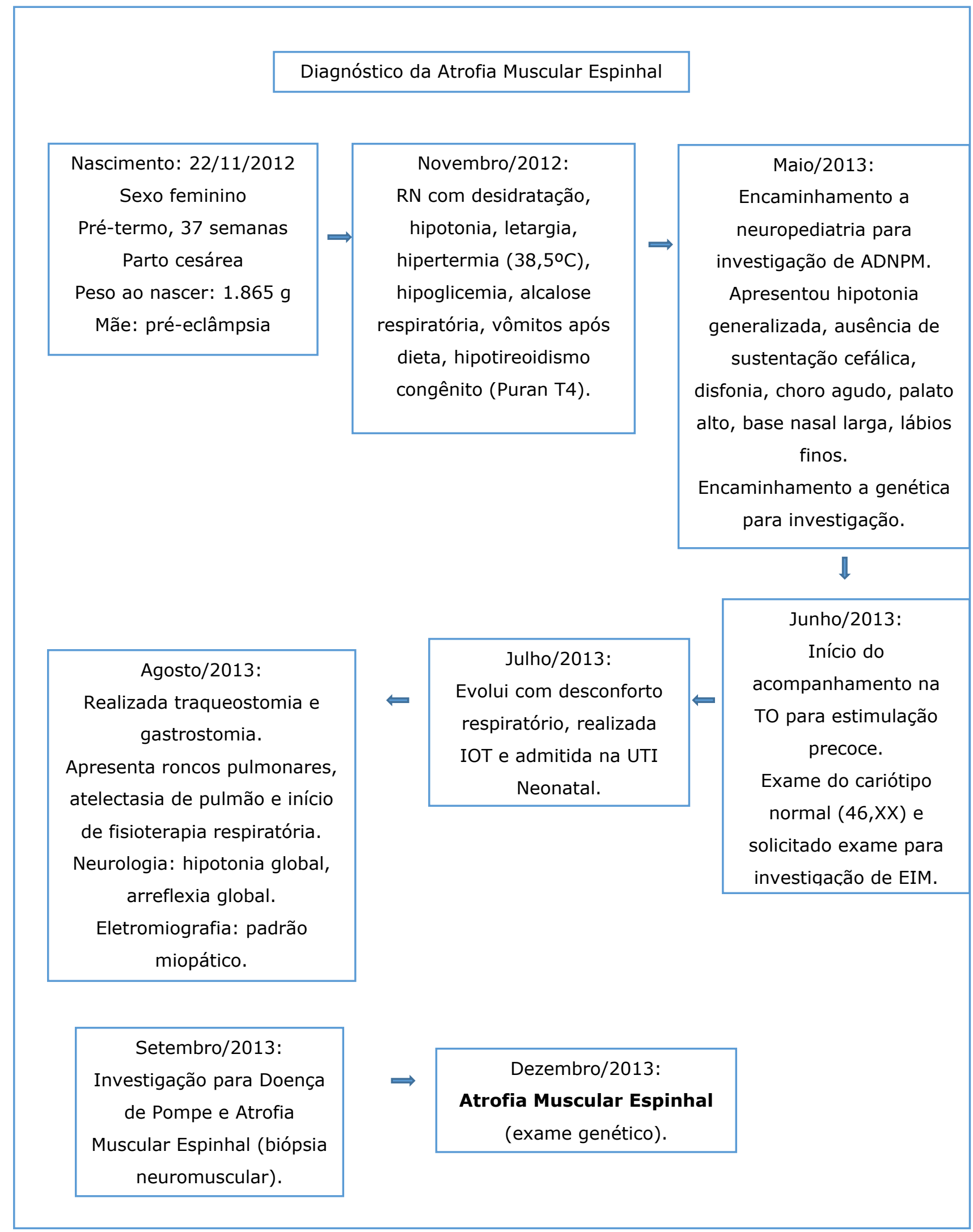

RN: recém-nascido; ADNPM: atraso no desenvolvimento neuropsicomotor; TO: terapia ocupacional; EIM: erros inatos do metabolismo; IOT: intubação orotraqueal; UTI: Unidade de Terapia Intensiva. 
Quadro 1. Cuidados multidisciplinares prestados à paciente desde sua internação.

\begin{tabular}{|c|c|}
\hline Especialidade & Cuidados prestados \\
\hline Enfermagem & $\begin{array}{l}\text { Anotação, evolução e prescrição de enfermagem sobre: aferição de sinais } \\
\text { vitais (SSVV), diurese, evacuações; exame físico; balanço hídrico; } \\
\text { administração de medicamentos e dieta; coleta de material e preparação } \\
\text { para exames e procedimentos cirúrgicos; aspiração de secreções; troca e } \\
\text { higiene dos materiais de aspiração; cuidados de higiene; cuidados com } \\
\text { oxigenoterapia, traqueostomia (TQT) e gastrostomia; auxílio nas atividades } \\
\text { instrumentais de vida diária (AIVDs), atividades do brincar e lúdicas. }\end{array}$ \\
\hline Fisioterapia & $\begin{array}{l}\text { Realização de fisioterapia respiratória e motora, como ventilação mecânica } \\
\text { por TQT; aspiração de TQT e vias áreas superiores; posicionamentos no leito } \\
\text { e para cuidados; mobilização passiva, global e alongamentos. }\end{array}$ \\
\hline Medicina & $\begin{array}{l}\text { Diagnóstico e conduta médica; pedido e avaliação de exames; prescrição de } \\
\text { medicação; procedimentos cirúrgicos; avaliação dos parâmetros } \\
\text { respiratórios; exame físico. }\end{array}$ \\
\hline Nutrição & $\begin{array}{l}\text { Responsável pela nutrição clínica: evolução de peso; estatura; classificação } \\
\text { de curva de crescimento; requerimentos nutricionais; prescrição de dieta e } \\
\text { conduta nutricional. }\end{array}$ \\
\hline Pedagogia & Realização de atividades educacionais. \\
\hline Psicologia & $\begin{array}{l}\text { Atendimentos de comunicação alternativa, informações aos pais sobre as } \\
\text { mudanças ocorridas, AIVDs e atividades lúdicas. }\end{array}$ \\
\hline Serviço Social & $\begin{array}{l}\text { Acompanhamento, apoio e orientação a família sobre solicitação do Benefício } \\
\text { de Prestação Continuada. }\end{array}$ \\
\hline $\begin{array}{l}\text { Terapia } \\
\text { ocupacional }\end{array}$ & $\begin{array}{l}\text { Atendimento de comunicação alternativa, AIVDs, atividades do brincar e } \\
\text { lúdicas, estimulação precoce, estimulação sensório-motora, proprioceptiva, } \\
\text { adequação postural e movimentação passiva; massagem proprioceptiva. }\end{array}$ \\
\hline
\end{tabular}

\section{DISCUSSÃO}

No presente trabalho, descrevemos uma paciente com AME tipo $1 \mathrm{~B} / \mathrm{C}$. De acordo com o Protocolo Clínico e Diretrizes Terapêuticas (PCDT) da Atrofia Muscular Espinhal $5 q$ tipo 1, aprovado em 2019, essa doença pode ser subdivida em tipos $1 \mathrm{~A}$ (uma cópia de SMN2) e 1B/C (duas a três cópias de SMN2), sendo que o número de cópias de SMN2 é responsável pela predição da gravidade da doença. 
No tipo $1 \mathrm{~A}$ a doença se inicia no período pré-natal, não há marco do desenvolvimento e a morte ocorre em semanas após o nascimento. Por outro lado, no 1B/C o início dos sintomas ocorre antes dos seis meses, o controle cefálico é pobre ou ausente, com expectativa de vida de até 24 meses $^{1}$.

Um estudo semelhante descreveu o caso de um menino de 2 anos e 10 meses de vida com diagnóstico genético de AME tipo I que exibiu no seu primeiro mês de vida dificuldade de sustentação de membros superiores, inferiores e região cervical, bem como recorrentes infecções respiratórias. Foi internado com seis meses de vida e desde então, recebe cuidados de rotina da UTI como, cabeceira elevada a 300, colchão anti-escaras, aferição dos sinais vitais a cada seis horas, fisioterapia respiratória e motora duas vezes ao dia e exercícios com fonoaudiólogos uma vez ao dia. Ele também recebia alimentação via gastrostomia e fazia uso de ventilação artificial via traqueostomia ${ }^{6}$. Já Vieira e colaboradores ${ }^{7}$ descreveram outro paciente com AME tipo I, que recebeu alta hospitalar e iniciou acompanhamento domiciliar com treinamento de sua família para realizar as manobras básicas para atendimento diário. Esse paciente recebia visitas periódicas de pediatras, terapeuta ocupacional e fisioterapeuta e, posteriormente foi assistido por equipe multidisciplinar completa, após a implantação do Programa de Internação Domiciliar no seu município de residência. Vale destacar que, o paciente com AME tipo I descrito nesse relato de caso encontra-se 
hospitalizado como aquele descrito por estudo prévio ${ }^{6}$ e contrário a outra pesquisa7. No entanto, nas três situações ficou evidente a importância de cuidados multidisciplinares constantes para melhora do estado geral e sobrevida dos pacientes com essa condição genética.

Um estudo descreveu 12 pacientes com AME, sendo eles sete com tipo I, um com tipo II e quatro com tipo III $^{8}$. Esses pacientes foram diagnosticados no período de janeiro de 2007 a outubro de 2009, com idades compreendidas entre os zero meses e os 21 anos de idade e o início dos sintomas variou de zero a seis meses de idade. Dos pacientes com AME tipo I, seis faleceram (idade mínima: quatro meses e máxima: 24 meses) e as causas das mortes foram insuficiência respiratória e parada cardiorrespiratória ${ }^{8}$. Diferentemente desses achados, 0 presente relato de caso mostrou uma sobrevida maior do que o esperado, talvez em virtude da assistência multiprofissional ofertada. Essa hipótese pode ser corroborada por estudo de 2014 que descreveu três crianças com AME tipo I e duas com o tipo II, com idade mínima de um ano e máxima de cinco anos, internadas na UTI pediátrica de Teresina, Piauí 9 . Elas superaram a expectativa de vida preconizada pela literatura, em função da atuação da equipe multiprofissional no atendimento a esses pacientes. Todos os pacientes exibiram alterações ortopédicas, sofreram intervenção cirúrgica do tipo traqueostomia e quatro deles, a gastrostomia. Os autores concluem que a AME tipo I é uma doença com prognóstico 
ruim e exige da equipe multiprofissional medidas de suporte, como ventilação mecânica, alimentação por sonda, administração de drogas e cuidados especiais individualizados. Outro achado interessante dessa pesquisa mostrou que as mães de crianças diagnosticadas com AME relataram que os movimentos fetais foram diminuídos no período gestacional e os sinais clínicos para internação hospitalar dessas crianças foi o comprometimento nos marcos de desenvolvimento, como não engatinharam no tempo adequado, não se sentaram, não tinham controle cefálico, não apresentaram fasciculações na língua e alguns apresentaram engasgo ocasional ${ }^{9}$.

Outro estudo, realizado no Chile, analisou 92 pacientes com AME, sendo que 23 apresentavam o tipo I, seis deles morreram com uma idade média de oito meses, 69,6\% foram traqueostomizados e alimentados por gastrostomia. 10 Segundo os autores, a AME tipo I é sub-representada, provavelmente devido às restrições de acesso ao diagnóstico precoce e à alta e precoce taxa de mortalidade ${ }^{10}$. Outra pesquisa recente descreveu 111 pacientes com AME tipo I, durante o período de 19792015. 11 Em dezembro de 2015, 95 pacientes haviam falecido, 13 estavam vivos e 3 perderam 0 acompanhamento. $\mathrm{O}$ uso de medidas de suporte (traqueostomia e gastrostomia) foi associada a uma sobrevida melhorada, enfatizando o impacto positivo das medidas de suporte na sobrevivência ${ }^{11}$, conforme demonstrado em nosso estudo. 
Atualmente, o nusinersena (Spinraza, Biogen Brasil Produtos Farmacêuticos Ltda.) é utilizado para o tratamento de AME tipo I e foi incorporado ao Sistema Único de Saúde (SUS) em abril de 201912,13. No entanto, trata-se de um medicamento de alto custo e com critérios específicos para o seu uso ${ }^{1}$. Ressalta-se que a paciente não faz uso deste medicamento.

O paciente com AME tipo I necessita de cuidados especiais que podem estacionar a progressão da doença e consequentemente, prolongar a sobrevida. Dentre esses cuidados a fisioterapia exerce um papel de suma importância no que diz respeito a manter a função respiratória e melhora na força muscular ${ }^{14}$. Uma revisão da literatura recente mostrou que as principais intervenções fisioterapêuticas utilizadas na AME nos últimos 10 anos foram exercícios em plataformas vibratórias, exercícios de fortalecimento com resistência, exercícios aquáticos e exercícios respiratórios ${ }^{15}$.

Vale ressaltar a importância dos PCDTs, documentos que orientam os profissionais de saúde sobre as condutas clínicas e terapêuticas, disponível atualmente para 36 doenças raras, dentre elas a AME, garantindo assim a assistência integral aos pacientes ${ }^{1}$.

\section{CONCLUSÃO}

O presente relato de caso descreve uma paciente com AME tipo I e evidencia a importância da assistência multidisciplinar em pacientes com essa condição genética, 
pois ela possibilita um aumento de sua sobrevida e melhora sua qualidade de vida.

\section{REFERÊNCIAS}

1.Brasil, Ministério da Saúde. Portaria Conjunta n¹5, de 22 de Outubro de 2019. Protocolo Clínico e Diretrizes Terapêuticas Atrofia Muscular Espinhal 5q Tipo 1. Brasília, DF, 2019. Disponível em: http://conitec.gov.br/images/Relatorios/2019/Relatorio PCDT AME.p df

2.Chrun LR, Costa LRC, Miranda GS, Almeida FM. Atrofia muscular espinhal tipo I: aspectos clínicos e fisiopatológicos/Spinal muscular atrophy type I: clinical and pathophysiological aspects. Rev Med (São Paulo) 2017;96:281-6. http://dx.doi.org/10.11606/issn.16799836.v96i4p281-286

3.Bozorg Qomi S, Asghari A, Salmaninejad A, Mojarrad M. Spinal Muscular Atrophy and Common Therapeutic Advances. Fetal Pediatr Pathol 2019;38:226-38.

http://dx.doi.org/10.1080/15513815.2018.1520374

4. Mercuri E, Pera MC, Scoto M, Finkel R, Muntoni F. Spinal muscular atrophy - insights and challenges in the treatment era. Nat Rev Neurol 2020;16:706-15. http://dx.doi.org/10.1038/s41582-02000413-4

5. McMillan HJ, Gerber B, Cowling T, Khuu W, Mayer M, Wu JW, et al. Burden of Spinal Muscular Atrophy (SMA) on Patients and Caregivers in Canada. J Neuromuscul Dis 2021;8:553-68. http://dx.doi.org/10.3233/JND-200610

6.Silva MCV, Przysiezny A, Capellani OJ. Síndrome de WerdnigHoffman (amiotrofia espinal do tipo 1): relato de caso. Arq Catarin Med 2013;42:96-9.

http://www.acm.org.br/revista/pdf/artigos/1220.pdf

7.Vieira LMN, Silva CAN, Oliveira MS, Pimenta LCA. O impacto do cuidado domiciliar na evolução da síndrome de Werdnig-Hoffmann: relato de caso. Rev Med Minas Gerais 2012;22:458-60. http://www.rmmg.org/artigo/detalhes/70

8.Pires M, Marreiros H, Francisco RC, Soudo A, Vieira JP. Atrofia muscular espinhal: análise descritiva de uma série de casos. Acta Med Port 2011;24(Suppl 2):95-102.

https://www.actamedicaportuguesa.com/revista/index.php/amp/artic le/viewFile/1522/1107

9. Feitosa WF, Silva MGP, Cunha KJB. Perfil de crianças com atrofia muscular espinhal em uma unidade de terapia intensiva pediátrica. Rev Interdiscipl 2014;7:173-82.

10.Alvarez K, Suarez B, Palomino MA, Hervias C, Calcagno G, Martínez-Jalilie $M$, et al. Observations from a nationwide vigilance program in medical care for spinal muscular atrophy patients in Chile. Arq Neuropsiquiatr 2019;77:470-7. http://dx.doi.org/10.1590/0004$\underline{282 \times 20190073}$ 
11.Ou SF, Ho CS, Lee WT, Lin $\mathrm{KL}$, Jones CC, Jong YJ, et al. Natural history in spinal muscular atrophy Type I in Taiwanese population: A longitudinal study. Brain Dev 2021;43:127-34. http://dx.doi.org/10.1016/j.braindev.2020.07.012

12.Caetano R, Hauegen RC, Osorio-de-Castro CGS. A incorporação do nusinersena no Sistema Único de Saúde: uma reflexão crítica sobre a institucionalização da avaliação de tecnologias em saúde no Brasil. Cad Saúde Pública 2019;35:e00099619. http://dx.doi.org/10.1590/0102-311X00099619

13. Domingos EM, Aguiar AM. O uso do Nusinersen no tratamento da Atrofia Muscular Espinhal: Revisão de literatura. Visão Acadêmica 2020;21:50-9.

https://revistas.ufpr.br/academica/article/download/70574/40703 14.Jorge MGAV, Carrapatoso BC, Fernandes ABS. A Fisioterapia na Amiotrofia Espinhal Progressiva Tipo I: Uma Revisão de Literatura. Rev Neurocienc 2013;21:402-7.

http://dx.doi.org/10.4181/RNC.2013.21.731.6p

15.Silva FS, Rodrigues JMP, Brito RN, Macedo TC, Borgmann AD. Intervenção Fisioterapêutica na Atrofia Muscular Espinhal: revisão de literatura.

Rev Neurocienc 2021;29:1-22.

https://doi.org/10.34024/rnc.2021.v29.11029 